\title{
Video Article \\ MRI Mapping of Cerebrovascular Reactivity via Gas Inhalation Challenges
}

\author{
Hanzhang Lu ${ }^{1}$, Peiying Liu ${ }^{1}$, Uma Yezhuvath ${ }^{1}$, Yamei Cheng ${ }^{1}$, Olga Marshall ${ }^{2}$, Yulin Ge \\ ${ }^{1}$ Advanced Imaging Research Center, University of Texas Southwestern Medical Center \\ ${ }^{2}$ Center for Biomedical Imaging, Department of Radiology, New York University School of Medicine
}

Correspondence to: Hanzhang Lu at hanzhang.lu@utsouthwestern.edu

URL: https://www.jove.com/video/52306

DOI: doi:10.3791/52306

Keywords: Medicine, Issue 94, Cerebrovascular reactivity, cerebrovascular diseases, MRI-compatible gas delivery systems, hypercapnia, hyperoxia, $\mathrm{CO}_{2}, \mathrm{O}_{2}$.

Date Published: $12 / 17 / 2014$

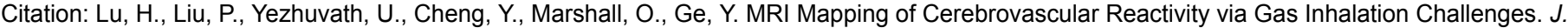
Vis. Exp. (94), e52306, doi:10.3791/52306 (2014).

\section{Abstract}

The brain is a spatially heterogeneous and temporally dynamic organ, with different regions requiring different amount of blood supply at different time. Therefore, the ability of the blood vessels to dilate or constrict, known as Cerebral-Vascular-Reactivity (CVR), represents an important domain of vascular function. An imaging marker representing this dynamic property will provide new information of cerebral vessels under normal and diseased conditions such as stroke, dementia, atherosclerosis, small vessel diseases, brain tumor, traumatic brain injury, and multiple sclerosis. In order to perform this type of measurement in humans, it is necessary to deliver a vasoactive stimulus such as $\mathrm{CO}_{2}$ and/or $\mathrm{O}_{2}$ gas mixture while quantitative brain magnetic resonance images (MRI) are being collected. In this work, we presented a MR compatible gas-delivery system and the associated protocol that allow the delivery of special gas mixtures (e.g., $\mathrm{O}_{2}, \mathrm{CO}_{2}, \mathrm{~N}_{2}$, and their combinations) while the subject is lying inside the MRI scanner. This system is relatively simple, economical, and easy to use, and the experimental protocol allows accurate mapping of CVR in both healthy volunteers and patients with neurological disorders. This approach has the potential to be used in broad clinical applications and in better understanding of brain vascular pathophysiology. In the video, we demonstrate how to set up the system inside an MRI suite and how to perform a complete experiment on a human participant.

\section{Video Link}

The video component of this article can be found at https://www.jove.com/video/52306/

\section{Introduction}

The brain represents about $2 \%$ of the total body weight, but consumes about $20 \%$ of the total energy ${ }^{1}$. Not surprisingly, sufficient and carefully regulated blood supply is critical to meet this high energy demand and for the brain to function properly. Furthermore, brain is a spatially heterogeneous and temporally dynamic organ, with different regions requiring different amount of blood supply at different time. Therefore, dynamic modulation of blood supply represents an important requirement in human brain circulation. Fortunately, it is known that blood vessels are not just rigid pipes and that an important function of blood vessel is to dilate and constrict based on the demand of the brain and physiologic conditions ${ }^{2}$.

This functional property of the vessel, known as Cerebral-Vascular-Reactivity (CVR), is thought to be a useful indictor of vascular health and may

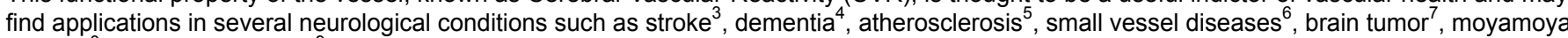
disease $^{8}$, and drug-addiction ${ }^{9}$. In physiology and anesthesiology literature, it is known that, because $\mathrm{CO}_{2}$ gas is a potent vasodilator, $\mathrm{CVR}$ can be assessed by altering the arterial $\mathrm{CO}_{2}$ level (e.g., inhalation of a small amount of $\mathrm{CO}_{2}$ ) while monitoring vascular responses ${ }^{10-13}$. In the imaging and radiology field, CVR mapping using MRI is rapidly emerging as a new marker of interest for many basic scientists and clinicians ${ }^{8,14-19}$. It is usually estimated by examining how much vascular response is induced by a vasoactive challenge. However, there is a need for technical advances in the gas delivery system and standardization of experimental protocol. Delivering special gas mixture to a subject inside the MRI scanner is not trivial and special considerations are required for an MRI-compatible design. Special considerations are required in designing MRI-compatible gas delivery system. These special considerations include: 1) all components must be non-metallic (metal cannot be used inside MRI); 2) the system should work within a small space that the MRI system and its head coil allow; 3 ) the system should work with a lying-down position (as MRI scanner requires) instead of sitting up, with no discomfort; 4) relevant physiological parameters, such as end-tidal CO2 (EtCO2, an approximation of $\mathrm{CO} 2$ content in the arterial blood) and arterial oxygen saturation, should be recorded accurately with seconds of timing accuracy and stored on a computer for analysis use. These issues may limit the scope of applications of CVR mapping.

In this report, we presented an experimental protocol that uses a comprehensive gas delivery system to modulate the content of inspired gas while the subject is lying inside the MRI scanner. Using this approach, the researcher can non-invasively apply a vasoactive stimulus to the participant with minimal discomfort or bulk motion. Physiological parameters and MRI images were recorded during the entire period of approximately $9 \mathrm{~min}$, which consisted of alternating blocks (1 min per block) of room-air and hypercapnic gas breathing. Representative results are presented. Potential applications and limitations are discussed. 


\section{Protocol}

NOTE: The protocol was approved by the University of Texas Southwestern Medical Center's Institutional Review Board.

\section{Diagram of the Gas Delivery System and Preparation Steps before the Experiment}

1. Review the diagram of the gas delivery system (Figure 1). Fill a $200 \mathrm{~L}$ Douglas bag (Item \#1) with a medical-grade gas mixture containing $5 \% \mathrm{CO}_{2}, 21 \% \mathrm{O}_{2}$, and $74 \% \mathrm{~N}_{2}$.

2. Place two diaphragms (Item \#4) in the two-way non-rebreathing valve (Item \#3) to ensure one way gas flow. Bring this assembled two-way valve and the gas-filled Douglas bag (Item \#1) into the magnet room.

3. Connect the gas delivery tube (Item \#7) to the input end of the two-way valve (Item \#3). Attach the gas delivery tube (Item \#7) to the side of the head coil for weight support. Connect the other end of the gas delivery tube (Item \#7) to the gas-filled Douglas bag (Item \#1).

4. Connect the mouthpiece (Item \#5) to the U-shape tube (Item \#12) via an elbow-connector (Item \#13 with gas sampling port sealed).

5. Connect the gas-sampling tubing (Item \#9) to the U-shape tube (Item \#12) via another elbow-connector (Item \#13).

6. Connect a small air-filter (Item \#11) to the other end of the gas-sampling tubing (Item \#9). Connect the other end of the air-filter (Item \#11) to the $\mathrm{CO}_{2}$ (Item \#14) monitor.

7. In the control room of the MRI suite, turn on $\mathrm{CO}_{2}$ (Item \#14) and pulse oximetry (Item \#15) monitors. Perform an auto-calibration for the $\mathrm{CO}_{2}$ monitor.

8. Connect the monitors to a laptop using USB ports. Open the HyperTerminal software that communicates with the monitors. Synchronize the monitors' times with a timer by writing down the timer time and corresponding monitors' times. The differences of the timer time and monitors' times will be accounted for in data processing (step 4.4).

9. Insert one end of a signaling bar into a waveguide so that one end of the bar is inside the magnet room and the other end is in the control room.

NOTE: The signaling bar is used for notifying the researcher inside the magnet room during the scan when switching of the three-way valve (Item \#2) is needed.

\section{Procedures during the Experiment}

1. Ask the subject to lie on the MRI table but do not put him/her into the magnet bore yet. Instruct the subject to press the nurse-call button if they feel discomfort during the scan. Ask the subject to wipe his/her nose with a piece of cleaning towelette to remove any grease.

2. Instruct the subject to breathe by mouth and to establish and maintain a breathing rhythm. Then apply a nose clip (Item \#6) on the subject.

3. Connect the open end of the U-shape tube (Item \#12) to the middle port of the two-way valve (Item \#3) via the elbow connector (Item \#13).

4. Gently place the mouthpiece into the subject's mouth so that the subject can breathe through the mouthpiece. Gently attach the finger sensor of the pulse oximetry (Item \#15) to the finger tip of the subject.

5. Insure that the head of the subject is in the iso-center of the head coil. Operate the MRI table to place him/her inside the magnet bore.

6. Ensure that one researcher stays inside the magnet room to monitor the subject and to be prepared to switch the three-way valve on the Douglas bag (Item \#1). Ensure that the researcher is wearing earplugs and a headset to block MRI noise.

7. Close the magnet room door and, in the control room, check the $\mathrm{EtCO}_{2}$ and arterial oxygen saturation fraction $\left(\mathrm{sO}_{2}\right)$ parameters displayed on the $\mathrm{CO}_{2}$ (Item \#14) and pulse oximetry (Item \#15) monitors. Start the recording of the parameters on the laptop.

8. Instruct the MRI operator to begin scanning using Blood-Oxygenation-Level-Dependent (BOLD) sequence. For 3T MRI scanner, the BOLD imaging parameters are: TR/TE $=1,500 / 30 \mathrm{msec}$, flip angle $=60^{\circ}$, field of view $=220 \times 220 \mathrm{~mm}^{2}$, matrix $=64 \times 64,29$ slices, thickness $=5 \mathrm{~mm}$, no gap between slices, 361 volumes. Review a pre-prepared sheet on which the timing of the valve switching is listed and gently swing the signaling bar when a switch is needed. Pay close attention to the subject's physiology, including heart rate, $\mathrm{sO}_{2}$, and $\mathrm{EtCO}_{2}$.

9. Now, inside the magnet room, switch on the Douglas bag (Item \#1) based on the movement of the signaling bar which controls the type of gas that the subject inspires.

10. Continue this procedure for the length of the study. During the 9 min imaging period, ensure that the valve switching takes place approximately once every minute. Note that the timing of the switch does not have to be exactly precise, as long as the EtCO2 time course is recorded.

NOTE: If the subject presses the nurse-call button during the scan, the scan will be aborted and the subject will be moved out of the magnet bore immediately. The researcher will remove the mouth piece and nose clip from the subject.

11. Use the intercom to notify the subject that the scan is completed. Pull the MRI table out. Gently remove the nose clip and mouthpiece from the subject while providing cleaning tissue to the subject to wipe any saliva. Gently remove the finger sensor of the pulse oximetry from the subject. The subject can then sit up and get off the MRI table.

\section{Clean-up Procedures after the Experiment}

1. Discard the gas sampling tubing (Item \#9), air-filter (Item \#11), mouthpiece (Item \#5) and nose clip (Item \#6).

2. Clean the reusable components. Disconnect the two-way valve (Item \#3) from the other components and remove the diaphragms (Item \#4) from the valve. Soak the two-way valve (Item \#3), diaphragm (Item \#4) and U-shape tube (Item \#12) in a concentrated phosphate-free disinfectant, containing surfactants such as Bacdown detergent disinfectant in a container for $20 \mathrm{~min}$. The dilution ratio of the detergent disinfectant and distilled water is 1:64.

3. Rinse the items described in 3.2 thoroughly with distilled water.

4. Dry the U-shape tube (Item \#12) with compressed air. Place the two-way valve (Item \#3) and diaphragms (Item \#4) on a clear countertop and allow them dry naturally and completely.

5. Empty the Douglas bag. Put away the signaling bar and gray tube. 


\section{Data Analysis to Compute CVR Map}

1. Save MRI data in Dicom file format or any other vendor-specific format. Transfer the data to a lab computer and convert the data into volumeby-volume file series, in which each file contains a 3D volume (e.g., BOLD image) corresponding to one time point.

2. Pre-process the image data. Perform image pre-processing steps including realignment, normalization, and smoothing using a script that calls library functions provided by the software Statistical Parametric Mapping (SPM). See supplemental code file 1 for an example of the Matlab script.

3. Use a script to read the $\mathrm{CO}_{2}$ recording, correct the sampling tubing delay by shifting the time course by a pre-calibrated amount (e.g., 12 $\mathrm{sec}$ in this setup which is determined as the time difference between one breath to the mouth piece and the appearance of that breath on the $\mathrm{CO} 2$ recording), and extract EtCO2 which is the envelope (positive peaks) of the raw time series. See supplemental code file 2 for the Matlab script.

4. Based on the synchronizing timer, segment the $\mathrm{EtCO}_{2}$ data to keep only the recording from 25 sec prior to the first image acquisition to 100 $\mathrm{sec}$ after the last image acquisition. The $\mathrm{EtCO}_{2}$ time course is the input function to the vasculature and is used as independent variable in the later-described linear regression analysis.

5. Identify the physiological delay between $\mathrm{EtCO}_{2}$ (measured in lung) and MRI signal (measured in the brain) by calculating cross-correlation coefficient (CC) between these two time courses at varying time shifts. The shift value that yields higher CC is considered the optimal time.

6. The $\mathrm{EtCO}_{2}$ time course is shifted by the optimal delay and only the time points matching those of MRI signal are preserved, resulting in a time series that are of the same length as the MRI signal.

7. Conduct a voxel-by-voxel linear regression using SPM in which the shifted EtCO2 time course is the independent variable and the MRI signal time course is the dependent variable.

8. Compute voxel-by-voxel map of CVR

$$
\operatorname{CVR}(\mathrm{i}, \mathrm{j}, \mathrm{k})=\frac{\beta 1(\mathrm{i}, \mathrm{j}, \mathrm{k})}{\beta 0(\mathrm{i}, \mathrm{j}, \mathrm{k})+\min (\mathrm{EtCO} 2) \cdot \beta 1(\mathrm{i}, \mathrm{j}, \mathrm{k})}
$$

where $(\mathrm{i}, \mathrm{j}, \mathrm{k})$ is the voxel index, $\beta 1$ is the regression coefficient associated with EtCO2 and $\beta 0$ is the regression coefficient associated with the constant term. $\min \left(\mathrm{EtCO}_{2}\right)$ is the minimal value of $\mathrm{EtCO}_{2}$ in the time course.

\section{Representative Results}

Two types of data are collected with the proposed protocol, physiological recordings and MR images. Figures 2 and $\mathbf{3}$ show recordings of physiological parameters from a representative subject. Black trace in Figure 2 displays the $\mathrm{CO}_{2}$ time course recorded by the $\mathrm{CO}_{2}$ monitor, which represents the $\mathrm{CO}_{2}$ content in air sampled near the mouthpiece. Note that this trace fluctuates rapidly as a function of time. This is because, during the inhalation phase of the breathing cycle, this recording reflects the $\mathrm{CO}_{2}$ content in the inhaling air and, during the exhalation phase, this recording reflects the $\mathrm{CO}_{2}$ content in the exhaled air. As such, the upper peak of each breathing cycle, referred to as end-tidal $\mathrm{CO}_{2}$ or $\mathrm{EtCO}_{2}$, represents the $\mathrm{CO}_{2}$ content in the lung, which can be approximately used as the $\mathrm{CO}_{2}$ concentration in the arterial blood. Note that $\mathrm{CO}_{2}$ concentration in the arterial blood is the driving force, i.e., input function, of the vasodilatory response. The peaks of the $\mathrm{CO}_{2}$ trace (red curve in Figure 2) were delineated with a detection algorithm which searches for the peak during each breath, in combination with manual inspection and correction. This was followed by a median filtering to remove abrupt peaks due to partial breath and to account for blood mixing during the course of flow from pulmonary vessels to cerebral vessels. The final $\mathrm{EtCO}_{2}$ time course is shown by the green curve in Figure 2 and it is used in the computation of CVR.

Figure 3 shows the time courses of breathing rate, arterial oxygen saturation fraction $\left(\mathrm{sO}_{2}\right)$, and heart rate. The breathing rate is obtained from the $\mathrm{CO}_{2}$ monitor while $\mathrm{sO}_{2}$ and heart rate are obtained from the pulse oximetry. As can be seen, these parameters do not show a systematic change with the hypercapnia challenge. Note that hypercapnia does cause hyperventilation in the subject, thus the $\mathrm{O}_{2}$ partial pressure in the lung will increase modestly. However, its impact on $\mathrm{sO}_{2}$ is minimal as blood hemoglobin is already largely saturated at room-air breathing and the oxygen dissociation curve is rather flat within that range.

Figure 4 shows representative BOLD MR images at different time of the experiment. The average signal intensity (in arbitrary MR units) is also shown. It can be seen that the BOLD signal in the brain shows an increase with $\mathrm{CO}_{2}$ inhalation. Note that the signal difference between room-air and $\mathrm{CO}_{2}$ periods is on the order of $1-3 \%$ in amplitude.

Combining data from physiological recordings and MR images, a voxel-by-voxel CVR map can be computed. Figure 5 shows representative CVR maps (in units of \% signal change per $\mathrm{mmHg} \mathrm{CO}_{2}$ change) of a healthy subject scanned on five different days, demonstrating an excellent reproducibility of the results. The proposed technique has so far been applied in studies of aging ${ }^{20}$, Alzheimer's Disease ${ }^{4}$, Multiple Sclerosis ${ }^{21}$, and exercise training 22 . 


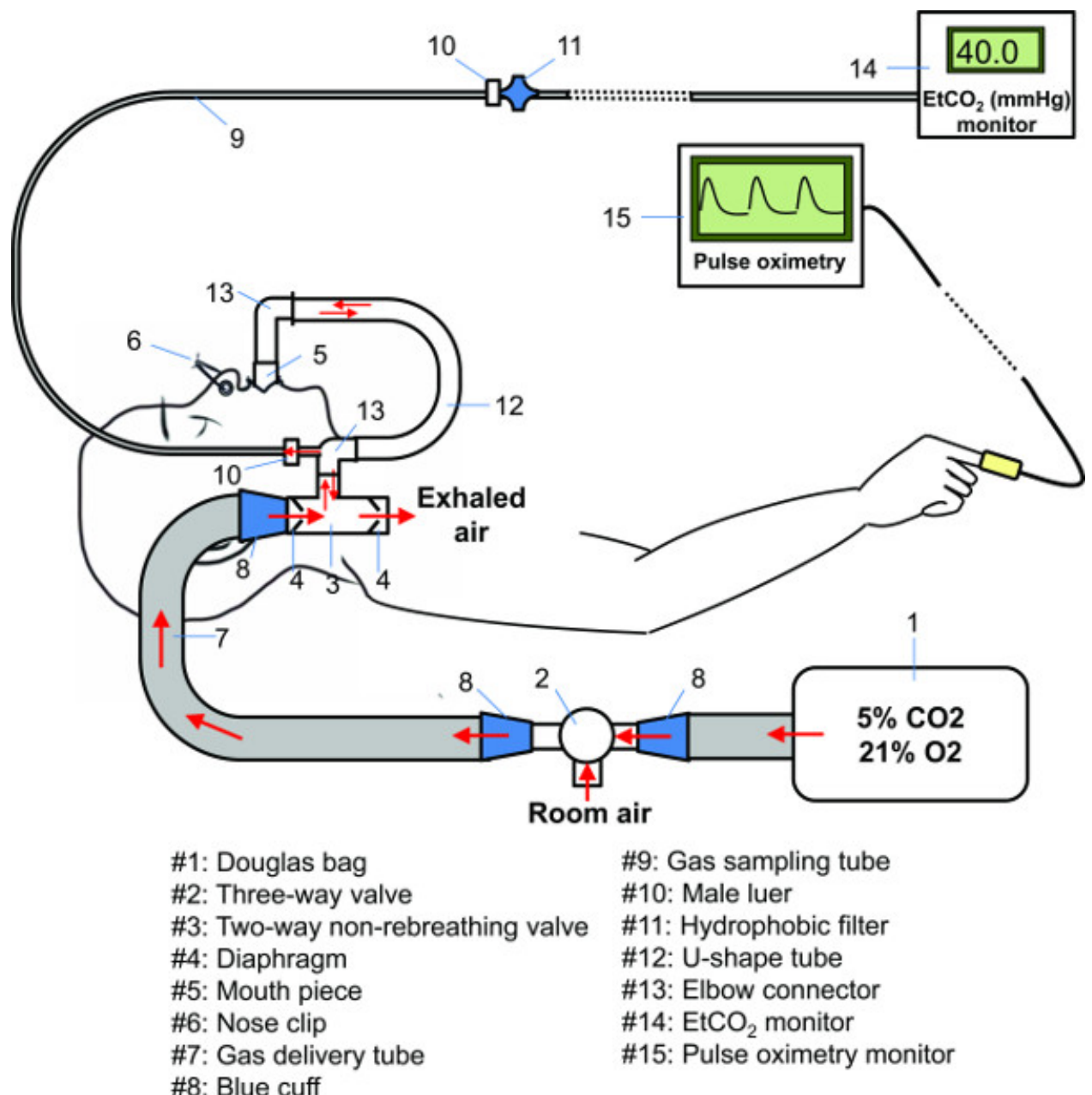

Figure 1. Diagram of the gas delivery system. Please click here to view a larger version of this figure. 


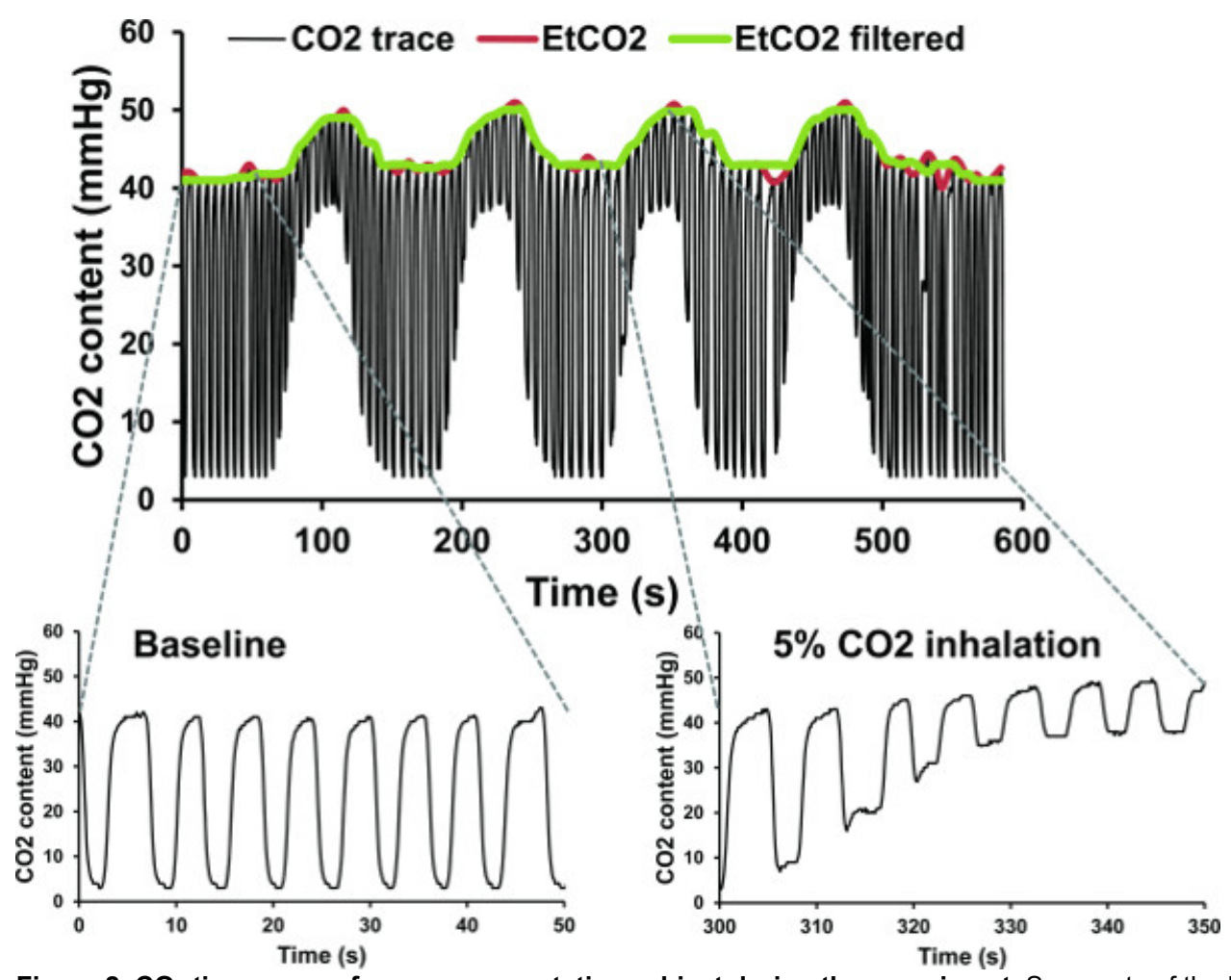

Figure 2. $\mathrm{CO}_{2}$ time course from a representative subject during the experiment. Segments of the breath-by-breath $\mathrm{CO}_{2}$ content trace as recorded by the $\mathrm{CO}_{2}$ monitor are shown for room air breathing period (lower left) and $5 \% \mathrm{CO}_{2}$ inhalation period (lower right). Extracted EtCO time courses are shown in colored curves. Please click here to view a larger version of this figure. 

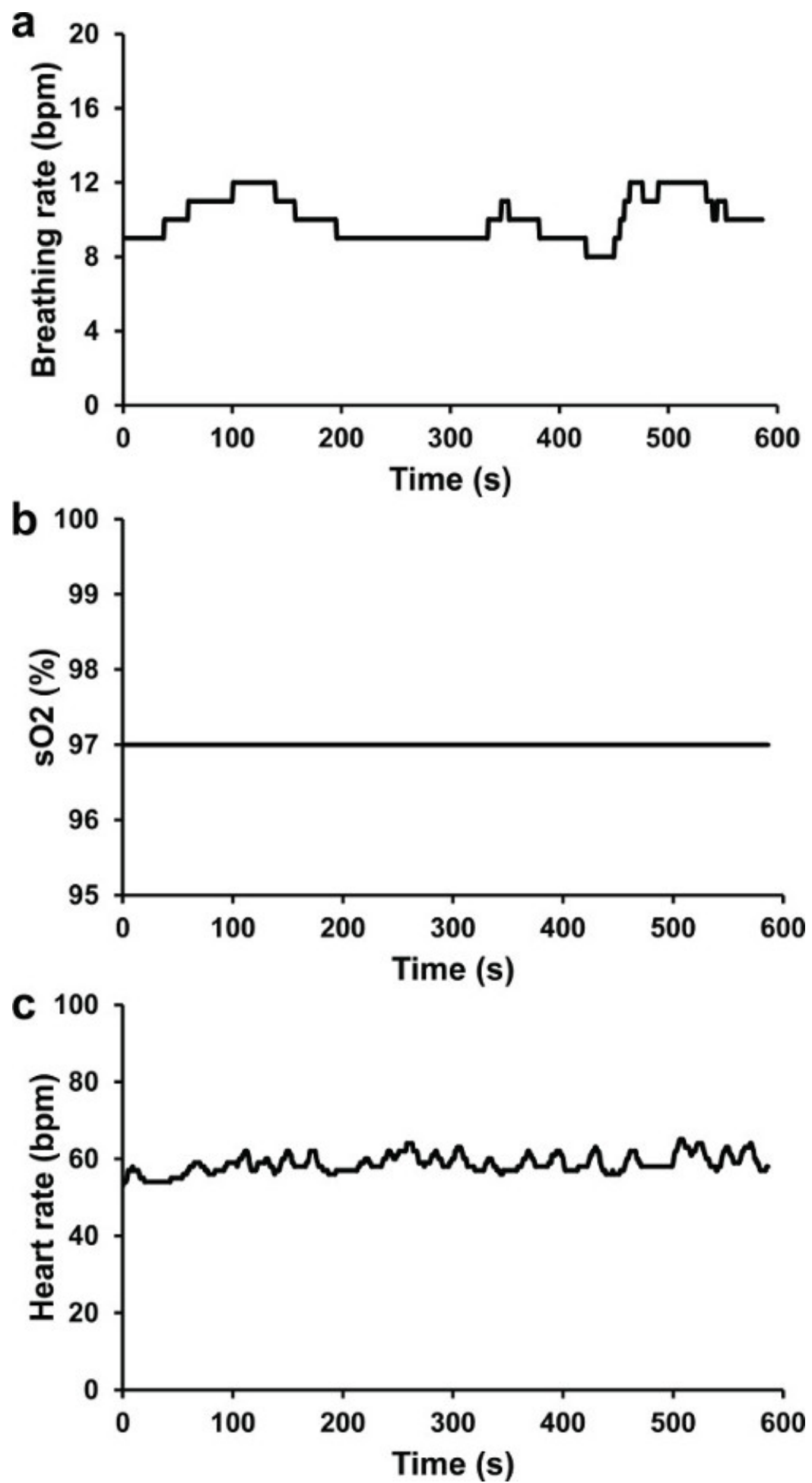

Figure 3. Recorded physiology parameters from a representative subject during the experiment. (A) Breathing rate (bpm, breath per minute) time course of the subject. (B) $\mathrm{sO}_{2}(\%)$ time course of the subject. (C) Heart rate (bpm, beat per minute) time course of the subject. The subject is the same one as that in Figure 1. Please click here to view a larger version of this figure. 


\section{Room air $\quad 5 \% \mathrm{CO} 2 \quad$ Room air $\quad 5 \% \mathrm{CO} 2$}

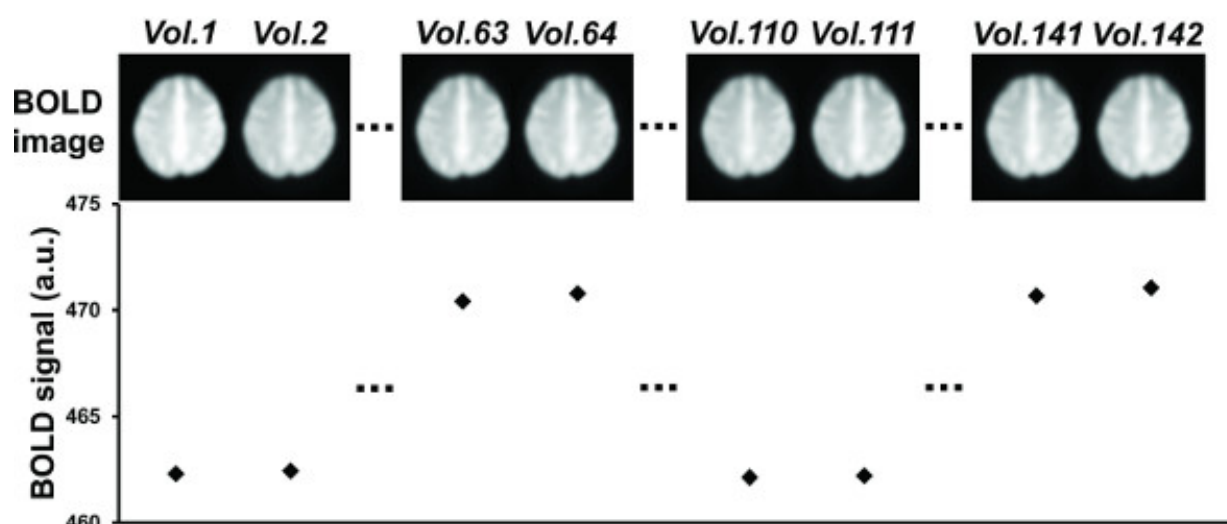

Figure 4. Representative BOLD MR images at different time of the experiment. Average signal intensities of the displayed brain slice (axial slice \#54 in MNI space) are showed in the bottom row. Please click here to view a larger version of this figure.

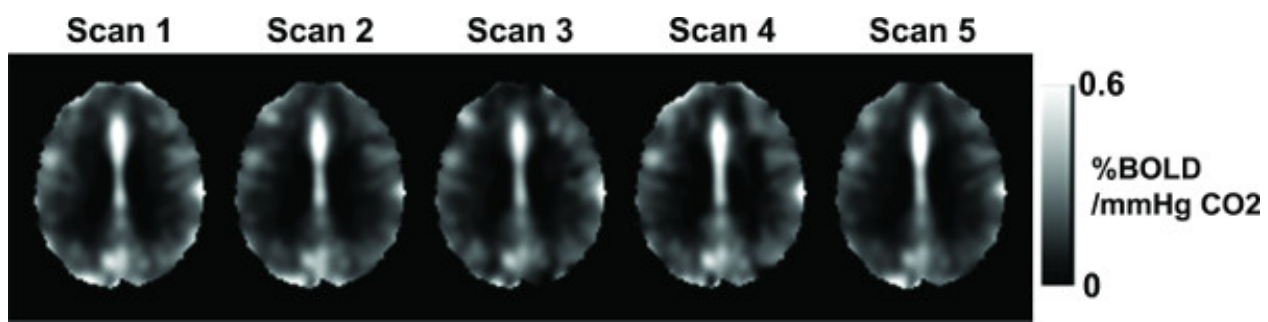

Figure 5. Representative CVR map from a representative subject. Please click here to view a larger version of this figure.

\section{Discussion}

This report presented an MR-compatible gas delivery system and a comprehensive experimental protocol that allows the mapping of vascular reactivity in the human brain. A diagram of the gas delivery system is illustrated in Figure 1. All parts inside the MRI scanner room are plastic to ensure their MRI compatibility. The system can be conceptually divided into three sub-systems, including a gas intake sub-system (bag, delivery tube, two-way valve), a breathing interface sub-system (nose clip, mouthpiece, U-shape tube), and a monitoring sub-system ( $\mathrm{CO}_{2}$ concentration, oxygen saturation, heart rate, breathing rate). The gas intake sub-system allows the gas to be inhaled to reach the two-way valve. Only inhaled air, but not exhaled air, will flow through this sub-system. The breathing interface sub-system allows the subject to breathe in and out the intended gas. Both inhaled and exhaled gas will flow through this sub-system. The monitoring sub-system should therefore sample the gas at a point along the breathing interface sub-system.

Clinical applications of this technique may include evaluations of brain vascular reserve in neurological diseases such as stroke, atherosclerosis, moyamoya disease, vascular dementia, multiple sclerosis, and brain tumor. The technique can also be used in functional MRI studies to normalize or calibrate fMRI signal for a better quantification of neural activity ${ }^{23,24}$.

An important feature of the proposed system and experimental protocol is that the gas mixture can be delivered to the subject while causing minimal motion or discomfort. Therefore, it is critical to place the U-shape tube (Item \#12) such that it (and the mouthpiece connected to the end of it) naturally falls downwards into the subject's mouth. This way, the subject does not need to use his facial muscle to hold or support the mouthpiece. It is also important to be aware that the subject will not be able to talk while the mouthpiece is in their mouth. Therefore, the researcher should avoid talking to the subject with a question tone. Instead, only clear, definitive instructions should be given. Additionally, a researcher should pay close attention to the physiological parameters (e.g., $\mathrm{EtCO}_{2}, \mathrm{sO}_{2}$, heart rate, breathing rate) during the entire course of the experiment and respond promptly when one or more of the physiological parameters deviate outside the typical range.

While an exhaustive survey of other gas delivery systems used in the literature is beyond the scope of this article, it is useful to compare the current system to a few commonly used ones ${ }^{17,18}$. A major difference is that our system uses a mouthpiece to deliver the intended gas while most other systems have used a mask in design. The potential complications of using a mask are two folds. First, a mask occupies a substantial amount of space, and it might not always be feasible to fit the mask into the tight space inside the head coil, considering that, for many subjects, their noses would almost touch the head coil even without a mask. This is especially the case for head coils intended to achieve high sensitivity, which are usually designed to fit tightly to the subject's head. A second complication associated with a mask design is that there is large space inside the mask which results in substantial mixing of inhaled and exhaled gas. Consequently, it could affect the accuracy of the measurement of $\mathrm{EtCO}_{2}$, which ideally should be based on exhaled gas only. Accurate $\mathrm{EtCO}_{2}$ is of course important for the reliability of the CVR map. Another major difference of our system in comparison to many other systems is that our system delivers the gas from a bag instead of a gas tank. Therefore, tanks are not needed in the scanner area, saving precious space in the control room of an MRI suite. In our design, we bring the bag before the start of the scan and, following the scan, the bag is emptied, folded, and put away. Finally, compared to several other systems ${ }^{18,21}$, the current gas delivery system is simpler, requires less training time, and its consumables are less expensive.

It should be pointed out that, although the protocol presented in this report has primarily focused on $\mathrm{CO}_{2}$ inhalation, the presented gas delivery system allows the delivery of other gas mixtures (e.g., any fraction of $\mathrm{O}_{2}$, any fraction of $\mathrm{CO}_{2}$, any fraction of $\mathrm{N}_{2}$, and their combination) to a 
human for them to breathe while s/he is lying inside the MRI scanner. One can also use the gas delivery system outside the context of MRI, for example in conjunction with electroencephalogram (EEG), magnetoencephalogram (MEG), positron emission tomography (PET), or optimal imaging.

When providing a recommendation of imaging parameters, we have primarily focused on BOLD sequence. Another sequence that can be potentially used in CVR mapping is Arterial Spin Labeling (ASL) MRI, which provides a quantitative measure of cerebral blood flow (CBF) in physiologic units ( $\mathrm{ml}$ blood per $100 \mathrm{~g}$ tissue per min). Therefore, the advantage of ASL-based CVR mapping is that the results are easier to interpret, unlike BOLD signal which reflects a combined effect of blood flow, blood volume as well as possible contributions of brain metabolic alterations during $\mathrm{CO}_{2}$ challenge ${ }^{25-27}$. However, a limitation of the ASL technique is that its sensitivity is several folds lower than that of BOLD ${ }^{28}$. As a result, our experience is that, at present, it is highly challenging to obtain an individual-level, voxel-by-voxel CVR map using ASL. Therefore, for application studies of CVR, we mainly use the BOLD sequence and thus also focus on this technique in our recommendations.

One limitation of the present method is that breathing through a mouthpiece with the nose blocked (by a nose clip) is not entirely natural and some subjects (especially patients) may perceive this as a source of discomfort. Breathing with the mouthpiece and nose clip may also exacerbate the feeling of claustrophobia. Additionally, the subject may experience dry mouth due to breathing by mouth only. Therefore, it is recommended that the researcher try his best to complete the experiment swiftly. Finally, it is important to note that, based on the authors' experience, the potential discomfort mentioned above is transient and will disappear as soon as the experiment is finished.

\section{Disclosures}

HL and PL are inventors of a provisional patent, owned by UT Southwestern Medical Center, that describes part of the gas delivery system.

\section{Acknowledgements}

This work was partly supported by grants from the National Institutes of Health (NIH), under grant numbers R01 MH084021, R01 NS067015, R01 AG042753, NS076588, NS029029-20S1, R21 NS078656; and from National Multiple Sclerosis Society, under grant number of RG4707A2.

\section{References}

1. Attwell, D., \& Laughlin, S. B. An energy budget for signaling in the grey matter of the brain. J Cereb Blood Flow Metab. 21 (10), $1133-1145$, doi:10.1097/00004647-200110000-00001, (2001).

2. Kety, S. S., \& Schmidt, C. F. The Effects of Altered Arterial Tensions of Carbon Dioxide and Oxygen on Cerebral Blood Flow and Cerebral Oxygen Consumption of Normal Young Men. J Clin Invest. 27 (4), 484-492, doi:10.1172/JCI101995, (1948).

3. Krainik, A., Hund-Georgiadis, M., Zysset, S., \& von Cramon, D. Y. Regional impairment of cerebrovascular reactivity and BOLD signal in adults after stroke. Stroke. 36 (6), 1146-1152, doi:10.1161/01.STR.0000166178.40973.a7, (2005).

4. Yezhuvath, U. S. et al. Forebrain-dominant deficit in cerebrovascular reactivity in Alzheimer's disease. Neurobiol Aging. 33 (1), 75-82, doi:10.1016/j.neurobiolaging.2010.02.005, (2012).

5. Mandell, D. M. et al. Mapping cerebrovascular reactivity using blood oxygen level-dependent MRI in Patients with arterial steno-occlusive disease: comparison with arterial spin labeling MRI. Stroke. 39 (7), 2021-2028, 10.1161/STROKEAHA.107.506709, (2008).

6. Greenberg, S. M. Small vessels, big problems. N Engl J Med. 354 (14), 1451-1453, doi:10.1056/NEJMp068043, (2006).

7. Hsu, Y. Y. et al. Blood oxygenation level-dependent MRI of cerebral gliomas during breath holding. J Magn Reson Imaging. 19 (2), 160-167, doi:10.1002/jmri.10447, (2004).

8. Mikulis, D. J. et al. Preoperative and postoperative mapping of cerebrovascular reactivity in moyamoya disease by using blood oxygen leveldependent magnetic resonance imaging. J Neurosurg. 103 (2), 347-355, (2005).

9. Han, J. S. et al. BOLD-MRI cerebrovascular reactivity findings in cocaine-induced cerebral vasculitis. Nat Clin Pract Neurol. 4 (11), 628-632, doi:10.1038/ncpneuro0918, (2008).

10. Ellingsen, I., Hauge, A., Nicolaysen, G., Thoresen, M., \& Walloe, L. Changes in human cerebral blood flow due to step changes in $P A O_{2}$ and $\mathrm{PACO}_{2}$. Acta Physiol Scand. 129 (2), 157-163, doi:10.1111/j.1748-1716.1987.tb08054.x, (1987).

11. Ide, K., Eliasziw, M., \& Poulin, M. J. Relationship between middle cerebral artery blood velocity and end-tidal $\mathrm{PCO}_{2}$ in the hypocapnichypercapnic range in humans. J Appl Physiol (1985). 95 (1), 129-137, 10.1152/japplphysiol.01186.2002, (2003).

12. Xie, A. et al. Cerebrovascular response to carbon dioxide in patients with congestive heart failure. Am J Respir Crit Care Med. 172 (3), 371-378, doi:10.1164/rccm.200406-8070C, (2005).

13. Rostrup, E. et al. Regional differences in the CBF and BOLD responses to hypercapnia: a combined PET and fMRI study. Neuroimage. 11 (2), 87-97, doi:10.1006/nimg.1999.0526, (2000).

14. Zande, F. H., Hofman, P. A., \& Backes, W. H. Mapping hypercapnia-induced cerebrovascular reactivity using BOLD MRI. Neuroradiology. 47 (2), 114-120, doi:10.1007/s00234-004-1274-3, (2005).

15. Kastrup, A., Kruger, G., Neumann-Haefelin, T., \& Moseley, M. E. Assessment of cerebrovascular reactivity with functional magnetic resonance imaging: comparison of $\mathrm{CO}(2)$ and breath holding. Magn Reson Imaging. 19 (1), 13-20 (2001).

16. Bright, M. G., Donahue, M. J., Duyn, J. H., Jezzard, P., \& Bulte, D. P. The effect of basal vasodilation on hypercapnic and hypocapnic reactivity measured using magnetic resonance imaging. J Cereb Blood Flow Metab. 31 (2), 426-438, doi:10.1038/jcbfm.2010.187, (2011).

17. Slessarev, M. et al. Prospective targeting and control of end-tidal $\mathrm{CO}_{2}$ and $\mathrm{O}_{2}$ concentrations. J Physiol. 581 (Pt 3), 1207-1219, doi:10.1113/ jphysiol.2007.129395, (2007).

18. Wise, R. G. et al. Dynamic forcing of end-tidal carbon dioxide and oxygen applied to functional magnetic resonance imaging. $J$ Cereb Blood Flow Metab. 27 (8), 1521-1532, doi:10.1038/sj.jcbfm.9600465, (2007).

19. Yezhuvath, U. S., Lewis-Amezcua, K., Varghese, R., Xiao, G., \& Lu, H. On the assessment of cerebrovascular reactivity using hypercapnia BOLD MRI. NMR Biomed. 22 (7), 779-786, doi:10.1002/nbm.1392, (2009). 
20. Lu, H. et al. Alterations in cerebral metabolic rate and blood supply across the adult lifespan. Cereb Cortex. 21 (6), 1426-1434, doi: 10.1093/ cercor/bhq224, (2011).

21. Marshall, O. et al. Impaired cerebral vascular reactivity in multiple sclerosis measured with hypercapnia perfusion MRI. JAMA Neurology In press (2014).

22. Thomas, B. P. et al. Life-long aerobic exercise preserved baseline cerebral blood flow but reduced vascular reactivity to $\mathrm{CO}_{2}$. J Magn Reson Imaging. 38 (5), 1177-1183, doi:10.1002/jmri.24090, (2013).

23. Liu, P. et al. Age-related differences in memory-encoding fMRI responses after accounting for decline in vascular reactivity. Neuroimage. 78 415-425, doi:10.1016/j.neuroimage.2013.04.053, (2013).

24. Liu, P. et al. A comparison of physiologic modulators of fMRI signals. Hum Brain Mapp. 34 (9), 2078-2088, doi:10.1002/hbm.22053, (2013).

25. Zappe, A. C., Uludag, K., Oeltermann, A., Ugurbil, K., \& Logothetis, N. K. The influence of moderate hypercapnia on neural activity in the anesthetized nonhuman primate. Cereb Cortex. 18 (11), 2666-2673, doi:10.1093/cercor/bhn023, (2008).

26. Xu, F. et al. The influence of carbon dioxide on brain activity and metabolism in conscious humans. J Cereb Blood Flow Metab. 31 (1), 58-67, doi:10.1038/jcbfm.2010.153, (2011).

27. Thesen, T. et al. Depression of cortical activity in humans by mild hypercapnia. Hum Brain Mapp. 33 (3), 715-726, doi:10.1002/hbm.21242, (2012).

28. Lu, H., Golay, X., Pekar, J. J., \& Van Zijl, P. C. Functional magnetic resonance imaging based on changes in vascular space occupancy. Magn Reson Med. 50 (2), 263-274, doi:10.1002/mrm.10519, (2003). 